This item was submitted to Loughborough's Research Repository by the author.

Items in Figshare are protected by copyright, with all rights reserved, unless otherwise indicated.

\title{
Bone geometry according to menstrual function in female endurance athletes
}

PLEASE CITE THE PUBLISHED VERSION

http://dx.doi.org/10.1007/s00223-013-9700-3

\section{PUBLISHER}

(C) Springer

VERSION

AM (Accepted Manuscript)

\section{LICENCE}

CC BY-NC-ND 4.0

\section{REPOSITORY RECORD}

Duckham, Rachel, Christine A. Bailey, Noel Cameron, Katherine S.F. Brooke-Wavell, Nicholas Peirce, and Gregory D. Summers. 2019. "Bone Geometry According to Menstrual Function in Female Endurance Athletes". figshare. https://hdl.handle.net/2134/15540. 
Bone geometry according to menstrual function in female endurance athletes

Duckham R.L ${ }^{1}$, Peirce $N^{2}$, Bailey C.A ${ }^{1}$, Summers $\mathrm{G}^{3}$, Cameron $\mathrm{N}^{1}$, Brooke-Wavell $\mathrm{K}^{1}$

\section{Institutional affiliation of authors:}

${ }^{1}$ School of Sport, Exercise and Health Sciences, Loughborough University, Loughborough, Leicestershire, LE11 3TU

${ }^{2}$ University Hospitals NHS Trust/English Cricket Board, Loughborough

${ }^{3}$ Derby Hospitals NHS Foundation Trust, Derby, United Kingdom

Corresponding Author: Rachel Duckham

Corresponding Author Address:

School of Sport, Exercise and Health sciences

Loughborough University

Leicestershire

LE11 3TU, UK

Telephone: $\quad$ +441509222749

Fax: $\quad+441509223940$

Email: $\quad$ r.l.duckham@lboro.ac.uk 


\begin{abstract}
Introduction: Athletes have higher bone mineral density (BMD) relative to non-athletes. In amenorrheic athletes BMD may be compromised by estrogen deficiency but it is unknown whether this is accompanied by structural differences. Purpose: To compare femoral neck bone geometry and density of a/oligomenorrheic athletes (AA), eumenorrheic (EA) athletes and eumenorrheic controls (EC). Methods: 158 women (70 endurance athletes and 88 controls) were recruited. Femoral neck BMD, section modulus (Z) and width were measured using dual x-ray absorptiometry (DXA). Menstrual function was assessed by questionnaire and classified as eumenorrheic ( $\geq 10$ periods/year) or a/oligomenorrheic ( $\leq 9$ periods/year). Results: 24 athletes were AA and 44 EA. Femoral neck BMD was significantly higher in EA than AA (8\% difference), and EC (11\%, difference) [mean (SE) 1.118 (0.015); 1.023 (0.020) and 0.999 (0.014) $\mathrm{g} \mathrm{cm}^{-2}$ respectively; $\left.p<0.001\right] . \mathrm{Z}$ was significantly higher in EA than EC (11\% difference), [EA: 667 (19), AA: 625 (21), and EC: 592 (10) cm³ $p<0.001]$. Femoral neck width did not differ between groups. All differences persisted after adjustment for height, age and body mass. Conclusion: The higher femoral neck Z and BMD in athletes, despite similar width, may indicate that exercise related bone gains are endosteal rather than periosteal Athletes with amenorrhea had smaller increments in bone mass rather than structural adaptation. The maintained femoral neck width in controls may be an adaptive mechanism to conserve bone strength in bending despite inactivity related bone decrement.
\end{abstract}

Keywords: Bone Geometry, Bone mineral Density, Menstrual dysfunction, Female, Endurance athletes

\title{
Mini Abstract
}

Low bone density (BMD) in estrogen deficient athletes may be accompanied by structural adaptations. Eumenorrhoeic athletes had greater BMD and section-modulus than sedentary controls, with amenorrheic athletes having intermediate values. All groups had similar femoral neck width, which may be an adaptive mechanism to conserve bone strength.

\section{Introduction}

Athletes have higher bone mineral density (BMD) than controls, but amenorrheic athletes have substantially lower BMD at the lumbar spine and femoral neck than their regularly menstruating peers (1-5). Estrogen deficiency in amenorrheic athletes may promote low bone mass (6), although the effect of estrogen deficiency on bone strength is unknown.

BMD has been used as a proxy for bone strength $(7,8)$. However bone strength encompasses the bone's architecture, geometry, cortical porosity, and tissue mineralization which cannot be individually identified in BMD measurements (9), although some of these factors may contribute to BMD. Therefore it is important to consider structural determinants of bone strength such as section modulus (Z), a measure of strength in bending. This can be calculated using bone densitometry in the form of hip structural analysis (HSA).

Exercise may affect structural parameters and have BMD independent influences on bone strength (10). Estrogen deficiency also seems to influence bone structure, based on the studies in animals and observations in 
postmenopausal women $(11,12)$. Exercise has previously been shown to enhance the bone's accrual on the periosteal (outer) bone surface, thus conferring greater resistance to bending, whereas estrogen may inhibit periosteal apposition (12). In eumenorrheic women, exercise may be a potential preventive measure for osteoporotic fractures, as the increased high impact loads can lead to increases in BMD relative to sedentary women $(13,14)$. However in amenorrheic athletes, estrogen deficiency is related to bone loss, raising concerns about increased risk of future osteoporosis $(11,12)$. The effect of estrogen deficiency on bone strength will, however, depend on the location of bone loss. It is possible that in amenorrheic athletes, the low BMD is accompanied by structural differences such as increased bone diameter and that would preserve $\mathrm{Z}$ and strength in bending (11). Conversely, if the BMD decrement is from periosteal surface, $\mathrm{Z}$ may be reduced to a greater extent than BMD. The effect of amenorrhea on bone strength thus depends upon the location of the bone decrement, and this is currently unknown.

Whilst it is accepted that exercise and estrogen are beneficial to bone density $(12,15)$ the effects of estrogen deficiency on bone structural parameters are in athletes are unknown. It is possible that differences in bone strength between amenorrheic and eumenorrheic athletes are different from those that would be predicted based upon BMD alone. Thus the aim of this study was to compare bone geometry and density of a/oligomenorrheic athletes, eumenorrheic athletes and eumenorrheic controls.

\section{Methods}

\section{Study design}

A cross-sectional design was used to compare bone status in female endurance athletes and sedentary controls according to menstrual function. BMD, bone mineral content (BMC), and body composition were assessed using dual X-ray absorptiometry (DXA). DXA was also used to estimate bone geometric parameters using the advanced hip structural analysis (AHA) software. Questionnaires were completed to assess current and past menstrual function. Bone parameters were compared between eumenorrheic controls with no history of menstrual dysfunction (EC) and athletes classified according to current menstrual function as eumenorrheic athletes (EA) or a/oligomenorrheic athletes (AA). To allow a comparison based upon cumulative exposure to estrogen deficiency, athletes were also classified according to menstrual history as regularly menstruating (RMA) or irregularly menstruating (IMA). Ethical approval was received from the National Research Ethics Service (NRES) and Loughborough University Ethics Committee. All participants gave written consent.

\section{Subjects}

68 female endurance athletes (57 runners and 11 triathletes) and 88 healthy sedentary controls aged between 1845 years were recruited. Athletes were recruited from sporting federations and registered running and triathlon clubs within the United Kingdom. They were required to be competing at an elite level for their age and/or training at a high volume (8-10 hours per week for a runner and 15-20 hours per week for a triathlete) in events from $800 \mathrm{~m}$ to the marathon or triathlon. Athletes were excluded if they had any injury that affected their training in the previous 12 months. Athletes and controls were excluded if they had been pregnant or lactating in the past 12 months; if they had commenced, ceased or changed hormonal contraception use within the previous 12 months or if they had any medical conditions or were taking medications which were likely to affect bone metabolism. The 88 controls were recruited within the Loughborough community for a previous intervention 
study, the aim of which was to determine the optimum weekly frequency of exercise to increase bone mass in premenopausal women who do not regularly participate in physical activity (16). Baseline measurements are reported here. Controls were screened to exclude individuals who had a body mass index $>30 \mathrm{~kg} / \mathrm{m}^{2}$, participated in high impact or weight bearing exercise more than $1 \mathrm{~h} /$ week, or were not regularly menstruating $(<10-13$ menstrual cycles per year) $(16)$.

\section{BMD and geometry}

DXA was used to measure BMD and BMC of the lumbar spine (L1-L4), and femoral neck. Bone geometric properties of the femoral neck were estimated using the AHA software (Lunar Prodigy, GE Healthcare, Madison, WI, U.S.A version encore 12.2) to determine cross-sectional area (CSA), Z, minimal neck width and strength index. Femoral strength index is the ratio of estimated compressive yield strength of the femoral neck to the expected compressive stress of a fall on the greater trochanter adjusted for the patient's age, height and weight. This is estimated from $\mathrm{Z}$ but incorporates other variables also $(17,18)$

\section{Menstrual function}

Questionnaires were used to assess current and past menstrual function and hormonal contraceptive use. Participants were classified into three groups: a/oligomenorrheic phenotype athletes (AA) ( $\leq 9$ periods per year), eumenorrheic athletes (including those taking hormonal contraception) (EA) ( $\geq 10$ periods per year), and eumenorrheic controls (EC). Participants who reported changes in hormonal contraception use in the previous 12 months were excluded from the study.

Fasted serum samples (not synchronised to menstrual cycle) were collected. A high prevalence of polycystic ovary syndrome (PCOS) has been reported in female athletes $(19,20)$ serum samples were analysed for antimullerian hormone (AMH) to differentiate hypothalamic amenorrhoea from PCOS and premature ovarian failure: AMH is elevated in PCOS bot does not vary over the menstrual cycle (21). Analysis was conducted using generation II ELISA kit (Beckman Coulter, High Wycombe, UK)). Any athlete identified as having elevated AMH values (> 67.1 pmol/L) was excluded from this study.

\section{Calculation of menstrual index in athletes}

Menstrual index was calculated using a the equation derived by Grimston et al (17), to provide a summary measure of menstrual history in female athletes, which may involve changes in menstrual function (amenorrhea, oligomenorrhea and amenorrhea) over a life span. Athletes were asked to record their age at menarche (M), the number of years they had been amenorrheic ( 0 - 3 periods per year) and oligomenorrheic ( 4 - 10 periods per year) since menarche. Using the equation below menstrual index (MI) was calculated:

$$
M I=\underline{11.5 \text { (no. yrs) }+7 \text { (no. yrs) }+1.5 \text { (no. yrs) }}
$$

$$
\mathrm{C}-\mathrm{M}
$$

MI represents the average menses per year over the entire length of menstruation, 11.5, 7 and 1.5 represent the annual menstrual frequency at midpoints of the menstrual categories eumenorrhea, oligomenorrhea and amenorrhoea respectively, $\mathrm{M}$ represents age at menarche, and $\mathrm{C}$ is the current age. 
Based on the MI categories derived by Grimston et al (22), athletes were categorized into two groups, athletes with a history of menstrual regularity $\mathrm{MI} \geq 10$ (RMA) and athletes with a history of menstrual irregularity MI < 10 (IMA).

\section{Anthropometric measures}

Height and body mass were assessed using standard protocols using a stadiometer and a beam balance scale respectively in all participants. Body composition in athletes was assessed using DXA.

\section{Statistical analysis}

Descriptive statistics (mean and standard errors) were used to characterize the sample. Analysis of variance (ANOVA) was used to compare means between menstrual function groups, with a Tukey’s HSD Post Hoc test determining which groups differed. An analysis of covariance (ANCOVA) was used to adjust for age, height, and body mass. All statistical analysis was carried out using SPSS 16.0 (SPSS Chicago Illinois, USA). The level of significance was set at $p$ value $<0.05$.

\section{Results}

The physical characteristics of the three groups can be found in table 1. Of the sixty-eight endurance athletes forty-four (65\%) were eumenorrheic and 24 (35\%) were currently a/oligomenorrheic ( $\leq 9$ periods per year). None of the AA athletes had elevated anti-mullerian hormone values which are indicative of PCOS (median (IQR) 20.7 (19.2) pmol/L). The total duration of reported amenorrhoea in the a/oligomenorrheic group was (mean (SD) 4.5 (4.9) years. Nineteen (43\%) of the eumenorrheic athletes were currently taking hormonal contraception. According to the MI, 26 (40\%) athletes, but none of the controls, had a history of irregular menstruation. Three athletes did not provide adequate information to determine menstrual history using the MI.

EC had significantly lower height, and greater age and weight than both athlete groups, whether athletes were classified according to either current menstrual function or menstrual history (Table 1). Physical characteristics of athletes did not differ significantly according to either menstrual function or menstrual history (Table 1). Controls had greater percent body fat (29.2 (6.4)\%) compared to both athlete groups. In the athletes, percent body fat did not differ significantly between eumenorrheic and amenorrheic athletes: mean (SD) being 17.7 (5.9) and 16.1 (5.4) \% respectively.

\section{Bone mineral density (BMD) and bone mineral content (BMC)}

Comparisons of BMD and BMC according to current menstrual function are shown in table 2. BMD of the femoral neck was significantly higher in EA than both AA and EC (by 8\% and 11\% respectively, $p<0.001$ ). Femoral neck BMC was also significantly higher $(12 \%, \mathrm{p}<0.001)$ in EA than the EC $\mathrm{s}$, although AA did not differ significantly from other groups (figure 1).

Lumbar spine BMD was $8 \%$ higher in the EC than the AA $(\mathrm{p}=0.005)$. There were no significant differences between the two athlete groups (mean (SE) AA: 1.092 (0.026), EA: 1.148 (0.020) ,p=0.369).

\section{Bone geometry}

Bone geometric properties estimated at the hip using DXA are summarised in table 2. Femoral neck CSA was significantly higher in EA compared to AA and EC ( $9 \%$ and $11 \%$ difference respectively, $p<0.001)$, similarly to 
the femoral neck BMD. Z was significantly higher in EA than EC (11\% difference, $p=0.001)$. The strength index of the bone was significantly higher in EA and AA than EC (23\% and 14\% differences respectively, $p<0.001$ ) (figure 1).

There were no significant differences in geometric properties (Z, strength index and minimal neck width) between AA and EA groups (\% differences, Z: 6\%, p=0.272, strength index: $9 \%, p=0.128$ and minimal neck width: $2 \% \mathrm{p}=0.655)$.

\section{BMD and bone geometry according to menstrual index (MI)}

To examine whether bone properties differed according to estrogen exposure across the reproductive period sixty-five of the athletes were re-grouped according to menstrual index. Three of the athletes were excluded from the analysis as they provided incomplete information. Results were generally similar to those from the current menstrual function analysis at the lumbar spine; with those athletes reporting a history of IMA (N=26, MI<10) having significantly lower lumbar spine (L1-L4) BMD than RMA athletes (9\% difference, $p=0.007$ ) and EC groups (11\% difference, $p<0.001)$. In contrast to the findings according to current menstrual function, lumbar spine (L1-L4) BMC was significantly lower in IMA than RMA athletes (6\% difference, $p=0.026$ ).

IMA athletes had significantly higher CSA (6\% difference, $p=0.045)$ and strength index (20\% difference, $p<$ 0.001) than EC group. Similarly RMA athletes had significantly higher femoral neck BMD (10\% difference, $\mathrm{p}$ $<0.001$ ), CSA (9\% difference, , $\mathrm{p}<0.001)$, Z (11\% difference, $p=0.002)$ and strength index (16\% difference, $p<0.001$ ) than EC, but in contrast to the findings according to current menstrual function femoral neck BMD and CSA did not differ significantly between the athlete groups (Table 2).

\section{Adjustments for differences in physical characteristics}

After adjustment for age, body mass and height, similar significant differences in BMD, BMC and bone geometric properties at the femoral neck according to current menstrual function persisted. When comparing groups according to menstrual history athlete groups now showed significantly higher BMD after adjustments for physical characteristics (IMA: 1.077 (0.24), RMA: 1.134 (0.019), EC: 0.985 (0.013) g cm $\left.{ }^{-2}, \mathrm{p}<0.05\right)$, BMC (IMA: 5.0 (0.1), RMA: 5.2 (0.1), EC: 4.8 (0.1) g, p<0.05) and CSA (IMA: 157 (4), RMA: 162 (3), EC: 150(4) $\left.\mathrm{mm}^{2}, \mathrm{p}<0.05\right)$ at the femoral neck than EC. At the lumbar spine, differences in BMD according to current menstrual function were no longer significant but RMA athletes still had a significantly higher BMD at the lumbar spine compared to IMA athletes.

\section{Discussion}

This study is novel in that it compares bone geometry according to menstrual function in female endurance athletes and sedentary controls. A/oligomenorrheic athletes had lower BMD compared to eumenorrheic athletes but this was still higher than the eumenorrheic controls. Section modulus showed similar magnitude of differences between groups to BMD. Femoral neck width was similar in all groups, suggesting that the higher BMD and BMC in athletes is related to additional endosteal or trabecular bone, rather than periosteal apposition.

Menstrual dysfunction was prevalent in this sample of athletes with twenty four (35\%) of the athletes reporting current menstrual dysfunction and 40\% reporting a history of menstrual dysfunction when calculated as MI. These findings are consistent with previous reports which have indicated menstrual dysfunction is present in 
between 1 and 44\% (23) of athletes depending on the sport population surveyed and the definition of menstrual dysfunction $(23,24)$. Contrary to some previous findings $(25)$, there were no significant differences in height, body mass and age between the a/oligomenorrheic, and eumenorrheic athletes, although the sedentary control group were significantly older, heavier and shorter than the athlete groups.

Athletes had a significantly higher femoral neck BMD than the eumenorrheic sedentary controls in accordance with earlier studies (11, 13, 14, 23, 26). Torstveit and Sundgot-Borgen (26) found low BMD was three times more likely in non-athletes than in athletes, with athletes having 3-20\% higher BMD compared to non-athlete controls. In the present study eumenorrheic athletes had approximately 14\% higher BMD than controls after adjustments for age, height and weight. A/oligomenorrheic athletes had intermediate values; with mean BMD $8 \%$ lower than that in eumenorrheic athletes but $2 \%$ higher than that in controls. Prolonged exercise may thus partly counteract the skeletal effects of prolonged estrogen deficiency $(11,12,24)$. Similarly gymnasts who engage in a higher impact activity have greater BMD than normally active individuals despite later menarche and periods of amenorrhoea $(24,27)$.

The differences in BMD observed here may not entirely represent the differences in bone strength between groups, as exercise and estrogen deficiency may have structural effects that influence bone strength independently of BMD. This study is the first to examine the effects of amenorrhea on hip geometric parameters such as section modulus, related to strength in bending. Eumenorrheic athletes had significantly greater section modulus compared with eumenorrheic controls, which persisted after adjustment for weight, height and age. The decrement in section modulus in amenorrheiccompared to eumenorrheic athletes was of similar magnitude as that for femoral neck BMD (6\% vs 8\%) but was not statistically significant ( $\mathrm{p}=0.272)$. As with BMD, Z was not lower in amenorrheic athletes compared to regularly menstruating controls. A previous study in anorexic women with secondary amenorrhoea found that low BMD relative to controls was accompanied by lower structural properties (Z, cortical thickness), hence the bone's resistance to bending was compromised at the hip compared to healthy controls (28). The amenorrheic athletes in this study had a greater duration of amenorrhea than the anorexic women in the study of di Vasta et al (28), so the differences in BMD seem likely to be due to differences in skeletal loading, lean mass and possibly other nutritional or endocrine deficiencies that may be associated with anorexia.

There were no significant differences in femoral neck width between any of the groups although both exercise (11) and estrogen deficiency (29) have been suggested to enhance periosteal expansion. Exercise has been reported to increase bone diameters at the tibia and radius $(30,31)$, but bone width at the femoral neck did not differ between athletes engaging in different types of sports and controls (32). The discrepancy between studies may be explained by differences in loading between these skeletal sites, with more torsional loading at the tibia and radius. Previous findings in estrogen deficient women have been consistent with estrogen deficiency promoting periosteal expansion. In adolescents with anorexia, bone outer diameter was maintained despite a substantial decrement in bone density and cross-sectional area relative to controls (28). In postmenopausal women, periosteal diameter increased most rapidly in those with highest sex hormone binding globulin (hence presumably lower bio-available estradiol (33). However, differences were only $0.03 \mathrm{~mm} / \mathrm{year}$ so it is possible that duration of amenorrhoea and/or exercise in this study were not great enough to have yielded detectable differences in the femoral neck width. 
To have higher BMC and BMD at the femoral neck with the same bone width implies differences are related to either endocortical or trabecular bone. The fact that $\mathrm{Z}$ is higher in the athletes may make endocortical increments seem more likely. However, the study is cross-sectional so may be affected by selection bias. For runners, lightness offers a competitive advantage, so women with smaller skeletal size and mass may be more likely to succeed. It is possible that if athletes had not exercised, they would have had a smaller neck width than the controls consistent with lower body mass. It is therefore possible to suggest that exercise-associated periosteal apposition has counteracted an otherwise smaller neck width in athletes.

Although femoral neck BMD was significantly higher in athletes, lumbar spine BMD was highest in the sedentary controls, although differences were not significantly significant after adjustments for age, height and weight. Athletes with a history of amenorrhea had significantly lower spine BMD than their regularly menstruating peers, although there were no significant differences according to current menstrual function. Previous studies have reported lower lumbar spine BMD in amenorrheic than eumenorrheic athletes (13, 34-36). The lumbar spine may be more responsive to hormonal stimuli than the femoral neck due to the higher ratio of trabecular (62-70\%) to cortical bone (30-38\%) (23) and so theoretically may be more susceptible to estrogen deficiency related bone loss. That lumbar spine BMD differed according to menstrual index rather than current, amenorrhea, may suggest that cumulative estrogen exposure is most important. Another possible explanation is that many of the currently a/oligomenorrheic athletes in this study took part in resistance training, which may compensate for potential estrogen deficiency related losses in BMD at the lumbar spine by increased loading (26) and so may explain the preservation of the lumbar spine BMD in currently amenorrheic athletes in this study.

The limitations of this study include that bone geometric properties were estimated at the hip using AHA which only provides a 2-dimensional image in one plane. Differences in positioning of the femoral neck could result in changes in the measure of the geometric properties of the hip. In order to achieve a more accurate measure of bone geometry a 3-dimensional image would be preferable such as given by CT or pQCT. However in a sample of women of reproductive age there may be ethical concerns about the radiation dose required for hip CT measurement. Menstrual status was assessed retrospectively. Whilst amenorrhea is indicative of estrogen deficiency, we did not assess serum or urinary metabolites of estrogen throughout the menstrual cycle (24) which would have been demanding for participants and was beyond the scope of this investigation. The control group was not matched for age, height, and body mass to the athlete groups which would have been the optimal study design, as matching for body mass may offset the effects of exercise. However physical characteristics were added as covariates and findings were not substantially affected so the different characteristics probably did not contribute to findings.

The findings from this study may have implications for the clinical and applied sports medicine field illustrating that the skeletal effects of estrogen deficiency in amenorrheic athletes may be counteracted by loading induced bone gains. Even though these findings may indicate that amenorrheic athletes who participate in loading sports have some preservation of bone mineral density we cannot be sure that this adaptation is adequate to withstand the potentially higher levels of loading in this group. It is clear that the bone adaptations seen in eumenorrheic athletes are not as pronounced in a/oligomeonorrheic athletes therefore potentially conferring greater risk of bone dysfunction. Therefore it is important to continue to address oligomenorrhoea and amenorrhoea and its 
underlying causes in the athletic population. It can therefore be concluded from this study that eumenorrheic athletes had significantly higher femoral neck Z, as well as BMD, than controls. Amenorrheic athletes had intermediate values for both parameters, suggesting that amenorrhea limits exercise induced bone gains rather than inducing structural adaptation. Femoral neck width was very similar in all groups despite higher BMC in athletes; therefore, the additional bone in athletes may represent more endosteal bone, rather than periosteal apposition. The maintained femoral neck width in controls may be an adaptive mechanism to conserve bone strength in bending despite inactivity related bone decrement.

Funding: No external funding supported the work outlined in the manuscript.

\section{Disclosure of Interest: None}

\section{References}

1. Drinkwater BL (1994) 1994 C.H. McCloy Research lecture. Does physical activity play a role in preventing osteoporosis? Res Q Exerc Sport 65:197-206

2. Drinkwater BL, Nilson K, Chesnut CH, Bremner WJ, Shainholtz S, Southworth MB (1984) Bone mineral content of amenorrheic and eumenorrheic athletes. N Engl J Med 311:277-281

3. Myburgh KH, Bachrach LK, Lewis B, Kent K, Marcus R (1993) Low bone mineral density at axial and appendicular sites in amenorrheic athletes. Med Sci Sport Exerc 25:1197-1202

4. Micklesfield LK, Hugo J, Johnson C, Noakes TD, Lambert EV (2007) Factors associated with menstrual dysfunction and self reported bone stress injuries in female runners in the ultra- and half-marathons of the two oceans. Br J Sports Med 41:679-683

5. Grimston SK (1990) Menstrual, calcuim, and training history: relationship to bone health and female runners. Clin Sports Med 2:119-128

6. Rencken ML, Chesnut CH, Drinkwater BL (1996) Bone density at multiple skeletal sites in amenorrheic athletes. J Am Med Ass 276:238-240

7. Cummings SR, Black DM, Nevitt MC, Browner W, Cauley J, Ensrud K, Genant HK, Palermo L, Scott J, Vogt TM (1993) Bone density at various sites for prediction of hip fractures. Lancet 341:72-75

8. Hui SL, Slemenda CW, Johnston CC (1988) Age and bone mass as predictors of fracture in a prospective study. J Clin Invest 81:1804-1809

9. Bonnick SL (2007) HSA: beyond BMD with DXA. Bone 41:S9-S12

10. Jarvinen TLN, Kannus P, Pajamaki I, Vuohelainen T, Tuukkanen J, Jarvinen A, Sievanen H (2003) Estrogen deposits extra mineral into bones of female rats in puberty, but simultaneously seems to suppress the responsiveness of female skeleton to mechanical 
loading. Bone 32:642-651

11. Bass SL (2003) The structural adaptations of cortical bone to loading during different stages of maturation. J Musculoskelet Neuronal Interact 3:345-347

12. Saxon LK, Turner CH (2006) Low-dose estrogen treatment suppresses periosteal bone formation in response to mechanical loading. Bone 39:1261-1267

13. Bennell KL, Malcolm SA, Thomas SA, Reid SJ, Brukner PD, Ebeling PR, Wark D (1996) Risk factors for stress fractures in track and field athletes—a twelve-month prospective study. Am J Sports Med 24:810-818

14. Snow-Harter CM, Marcus R (1992) Exercise, bone mineral density and osteoporosis. Exerc Sport Sci Rev 7:1291-1296

15. Frost HM (1999) On the estrogen-bone relationship and postmenopausal bone loss: a new model. J Bone Miner Res 14:1473-1477

16. Bailey CA, Brooke-Wavell K (2010) Optimum frequency of exercise for bone health: randomised controlled trial of a highimpact unilateral intervention. Bone 46:1043-1049

17. Faulkner KG, Wacker WK, Barden HS, Simonelli C, Burke PK, Ragi S, Del Rio L (2006) Femur strength index predicts hip fracture independent of bone density and hip axis length.

Osteoporos Int 17:593-599

18. Yoshikawa T, Turner C, Peacock M, Selemenda C, Weaver C, Teegarden D, Markwardt P, Burr D (1994) Geometric structure of the femoral neck measured using dual energy X-ray absorptiometry. J Bone Miner Res 9:1053-1064

19. Rickenlund A, Carlstro“m K, Ekblom B, Brismar T, von Schoultz B, Hirschberg A (2004) Diurnal profiles of testosterone and pituitary hormones suggest different mechanisms for menstrual disturbances in endurance athletes. J Clin Endocrinol Metab 89:702-707

20. Rickenlund A, Carlstro“m K, Ekblom B, Brismar T, von Schoultz B, Hirschberg A (2003) Hyperandrogenicity is an alternative mechanism underlying oligomenorrhea or amenorrhea in female athletes and may improve physical performance. Fertil Steril 79: 947-955

21. Broekmans F, Visser J, Laven J, Broer S, Themmen A, Frauser B (2008) Anti-mullerian hormone and ovarian dysfunction. Trends Endocrinol Metab 19:340-347

22. Grimston S, Sanborn C, Miller P (1990) The application of historical data for evaluation of osteopenia in female runners: the menstrual index. Clin Sports Med 1990:108-118 
23. Bennell KL, Malcolm SA, Wark JD, Brukner PD (1997) Skeletal effects of menstrual disturbances in athletes. Scand J Med Sci Sports 7:261-273

24. Ducher G, Eser P, Hill B, Bass S (2009) History of amenorrhoea compromises some of the exercise-induced benefits in cortical and trabecular bone in the peripheral and axial skeleton: a study in retired elite gymnasts. Bone 45:760-767

25. Warren MP, Brooks-Gunn J, Fox RP, Holderness CC, Hyle EP, Hamilton WG (2002) Osteopenia in exercise-associated amenorrhea using ballet dancers as a model: a longitudinal study. J Clin Endocrinol Metab 87:3162-3168

26. Torstveit MK, Sundgot-Borgen J (2005) Low bone mineral density is two to three times more prevalent in non-athletic premenopausal women than in elite athletes: a comprehensive controlled study. Brit J Sport Med 39:282-287

27. Robinson TL, Snowharter C, Taaffe DR, Gillis D, Shaw J, Marcus R (1995) Gymnasts exhibit higher bone mass than runners despite similar prevalence of amenorrhea and oligomenorrhea. J Bone Miner Res 10:2635

28. DiVasta AD, Beck TJ, Petit MA, Feldman HA, LeBoff MS, Gordon CM (2007) Bone cross-sectional geometry in adolescents and young women with anorexia nervosa: a hip structural analysis study. Osteoporos Int 18:797-804

29. Kaptoge S, Dalzell N, Jakes RW, Wareham N, Day NE, Khaw KT, Beck TJ, Loveridge N (2003) Hip section modulus, a measure of bending resistance, is more strongly related to reported physical activity than BMD. Osteoporos Int 14:941-949

30. Haapasalo H, Kontulainen S, Sievanen H, Kannus P, Jarvinen M, Vuori I (2000) Exercise-induced bone gain is due to enlargement in bone size without a change in volumetric bone density: a peripheral quantitative computed tomography study of the upper

arms of male tennis players. Bone 27:351-357

31. Hamilton CJ, Swan VJD, Jamal SA (2010) The effects of exercise and physical activity participation on bone mass and geometry in postmenopausal women: a systematic review of pQCT studies. Osteoporos Int $21: 11-23$

32. Nikander R, Sievanen H, Heinonen A, Kannus P (2005) Femoral neck structure in adult female athletes subjected to different loading modalities. J Bone Miner Res 20:520-528

33. Ahlborg HG, Johnell O, Turner CH, Rannevik G, Karlsson MK 
(2003) Bone loss and bone size after menopause. N Engl J Med 349:327-334

34. Cobb KL, Bachrach LK, Greendale G, Marcus R, Neer RM, Nieves J, Sowers MF, Brown BW,

Gopalakrishnan G, Luetters C, Tanner HK, Ward B, Kelsey JL (2003) Disordered eating, menstrual irregularity, and bone mineral density in female runners.

Med Sci Sports Exerc 35:711-719

35. Hind K (2008) Recovery of bone mineral density and fertility in a former amenorrheic athlete. J Sport Sci Med 7:415-418

36. Christo K, Prabhakaran R, Lamparello B, Cord J, Miller KK, Goldstein MA, Gupta N, Herzog DB, Klibanski A, Misra M (2008) Bone metabolism in adolescent athletes with amenorrhea, athletes with eumenorrhea, and control subjects. Pediatrics 121:1127-1136 
Table 1: Physical Characteristics of athletes and sedentary controls according to current menstrual function (mean (SD)

\begin{tabular}{lccc}
\hline \hline & \multicolumn{3}{c}{ Current Menstrual Function } \\
& $\begin{array}{c}\text { EC } \\
(\mathrm{N}=88)\end{array}$ & $\begin{array}{c}\text { EA } \\
(\mathrm{N}=44)\end{array}$ & $\begin{array}{c}\text { AA } \\
(\mathrm{N}=24)\end{array}$ \\
\hline Age (years) & $32.8(8.4)$ & $26.9(7.9)^{*}$ & $23.9(6.4)^{*}$ \\
Body Mass (kg) & $61.8(10.2)$ & $55.4(6.6)^{*}$ & $55.4(4.9)^{*}$ \\
Height (m) & $1.63(0.10)$ & $1.66(0.10)^{*}$ & $1.68(0.05)^{*}$ \\
BMI (kg/m $\left.{ }^{2}\right)$ & $23.2(3.7)$ & $20.0(1.3) *$ & $19.6(1.5)^{*}$ \\
Body Fat (\%) & & $17.7(5.9)$ & $16.1(5.4)$ \\
Age at Menarche (yrs) & - & $13.9(0.2)$ & $14.5(0.1)$ \\
Training (hrs/week) & - & $12.8(4.7)$ & $13.1(4.9)$ \\
\hline \hline
\end{tabular}

*significantly different from EC ( $\mathrm{p}<0.05$ in ANOVA)

No significant differences between EA and AA

EC: Eumenorrheic control, EA: Eumenorrheic Athletes, AA: A/oligomenorrheic Athletes, 
Table 2: Comparisons of BMD, BMC and geometric measures in female athletes and sedentary controls according to current menstrual function and menstrual Index (MI): mean (SE)

\begin{tabular}{|c|c|c|c|c|c|}
\hline & \multirow{2}{*}{$\begin{array}{c}\text { Controls } \\
\text { EC } \\
(\mathrm{N}=88)\end{array}$} & \multicolumn{2}{|c|}{$\begin{array}{l}\text { Athlete Grouped according to } \\
\text { current Menstrual function }\end{array}$} & \multicolumn{2}{|c|}{$\begin{array}{l}\text { Athlete Grouped according to } \\
\text { Menstrual history (MI) }\end{array}$} \\
\hline & & $\begin{array}{c}\text { EA } \\
(\mathrm{N}=44)\end{array}$ & $\begin{array}{c}\text { AA } \\
(\mathrm{N}=24)\end{array}$ & $\begin{array}{c}\text { RMA } \\
(\mathrm{N}=39)\end{array}$ & $\begin{array}{c}\text { IMA } \\
(\mathrm{N}=26) \\
\end{array}$ \\
\hline \multicolumn{6}{|l|}{$\begin{array}{l}\text { Bone mineral density } \\
\text { (BMD) }\end{array}$} \\
\hline BMD Femoral Neck $\left(\mathrm{g} / \mathrm{cm}^{2}\right)$ & $0.999(0.014)$ & $1.118(0.015)^{\mathrm{a}}$ & $1.023(0.020)^{b}$ & $1.113(0.014)^{\mathrm{a}}$ & $1.059(0.022)$ \\
\hline BMD Spine (L1-L4) $\left(\mathrm{g} / \mathrm{cm}^{2}\right)$ & $1.188(0.014)$ & $1.148(0.020)$ & $1.092(0.026)^{\mathrm{a}}$ & $1.168(0.020)$ & $1.069(0.024)^{\mathrm{ac}}$ \\
\hline \multicolumn{6}{|l|}{$\begin{array}{l}\text { Bone mineral content } \\
(B M C)\end{array}$} \\
\hline BMC Femoral Neck (g) & $4.6(0.1)$ & $5.2(0.1)^{\mathrm{a}}$ & $4.8(0.1)$ & $5.1(0.1)$ & $5.0(0.1)$ \\
\hline BMC Spine (L1-L4) (g) & $61.4(1.1)$ & $62.0(1.7)$ & $57.7(1.9)$ & $61.4(1.5)$ & $56.3(1.8)^{\mathrm{c}}$ \\
\hline \multicolumn{6}{|l|}{$\begin{array}{l}\text { Femoral neck geometric } \\
\text { measures }\end{array}$} \\
\hline $\mathrm{CSA}\left(\mathrm{mm}^{2}\right)$ & $144(2)$ & $161(3)^{a}$ & $148(4)^{b}$ & $159(3)$ & $154(3)^{a}$ \\
\hline Minimum neck width (mm) & $28.5(0.2)$ & $28.4(0.3)$ & $28.9(0.5)$ & $28.4(0.4)$ & $28.4(0.4)$ \\
\hline Section modulus $(\mathrm{Z})\left(\mathrm{mm}^{3}\right)$ & $592(10)$ & $667(19)^{a}$ & $625(21)$ & $665(22)^{a}$ & $624(18)$ \\
\hline Strength Index & $1.61(0.03)$ & $2.06(0.0) 6^{\mathrm{a}}$ & $1.87(0.09)^{\mathrm{a}}$ & $1.94(0.06)^{\mathrm{a}}$ & $2.04(0.10)$ \\
\hline
\end{tabular}

${ }^{a}$ sig diff from EC, ${ }^{b}$ sig diff from EA, c sig diff from RMA, $\mathrm{p}<0.05$ ANOVA according to Tukey’s Post Hoc test

EC: Eumenorrheic control, EA: Eumenorrheic Athletes, AA: A/oligomenorrheic Athletes, RMA: Menstrual Index Regular Menstruating Athletes, IMA: Menstrual Index Irregular Menstruating Athlete. 
Figure 1 Femoral neck bone parameter mean differences for eumenorrheic (EA) and amenorrheic (AA) athletes from controls (mean difference and 95\% CI bars).

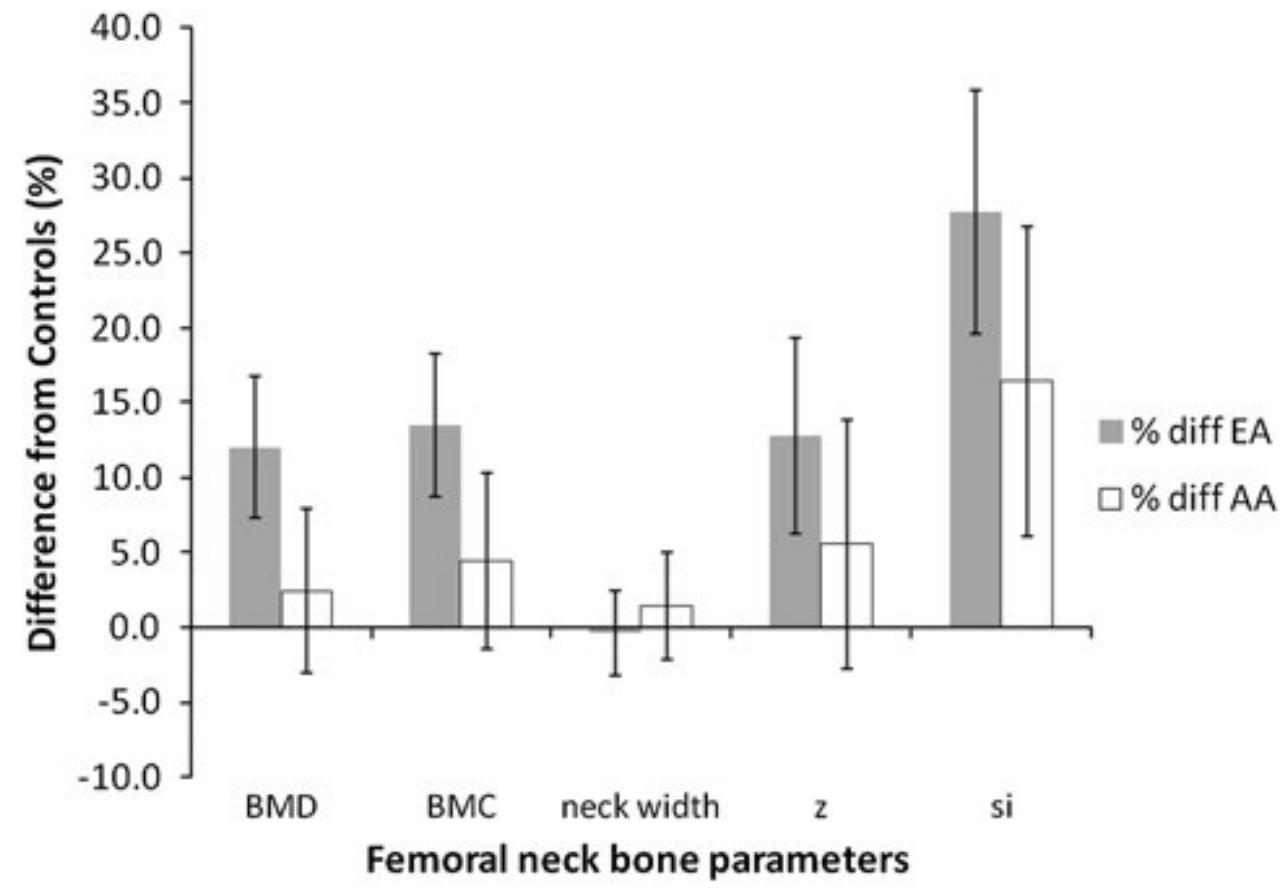

